\title{
Excision of the atrial Wilms' tumor thrombus without sternotomy, atriotomy and cardiovascular By-pass
}

\author{
Ali Tekin ${ }^{1}$, İsmail Yağmur ${ }^{1}$, Orkan Ergün², Mehmet Fatih Ayık ${ }^{3}$, Yüksel Atay ${ }^{3}$, \\ İbrahim Ulman ${ }^{1}$, Ali Avanoğlu ${ }^{1}$ \\ Departments of ${ }^{2}$ Pediatric Surgery, ${ }^{1}$ Division of Pediatric Urology, ${ }^{3}$ Pediatric Cardiovascular Surgery, Ege University \\ Faculty of Medicine, Izmir, Turkey.E-mail: dralitekin@gmail.com \\ Received: 9th May 2018, Revised: 4th September 2018, Accepted: 19th October 2018
}

SUMMARY: Tekin A, Yağmur İ, Ergün O, Ayık MF, Atay Y, Ulman İ, Avanoğlu A. Excision of the atrial Wilms' tumor thrombus without sternotomy, atriotomy and cardiovascular By-pass. Turk J Pediatr 2019; 61: 436-439.

The treatment of atrial-extention Wilms' tumor thrombus is surgical excision after chemotherapy. Atriotomy with cardiovascular by-pass is the one of the most common method for this procedure. Herein, we aimed to present a case of Wilms' tumor with a tumor thrombus extending into the right atrium totally excised with retrohepatic cavatomy.

A 3.5 year-old girl was admitted with the symptom of dysuria. The examinations revealed a mass consistent with Wilms' tumor in the middle and lower poles of the left kidney. Doppler ultrasound and Echocardiographic examinations showed a tumor thrombus extending into the right atrium and some pulmonary nodules which were interpreted to be metastasis. Wilms' tumor was histopathologically diagnosed by an open biopsy. After three courses of chemotherapy imaging studies revealed that the atrial axtention of the tumor thrombus persisted. The tumor thrombus was found to be fibrotic on the magnetic resonance imaging scan of the patient. Therefore, nephroureterectomy along with the excision of the tumor thrombus from the inferior vena cava was done with intraoperative continuous trans-esophageal echocardiography (TEE). The suprarenal and retrohepatic vena cava were exposed by dissecting and ligating all short hepatic veins and completely mobilizing the right lobe of the liver. The thrombus was dissected out via Vertical cavatomy at the retrohepatic level. TEE confirmed complete removal of the thrombus from the atrium; Vena cava was then repaired. There was no need for a blood transfusion, or cardiovascular by-pass (CPB) during the operation.

Total exposure of the retrohepatic and subdiaphragmatic vena cava using transplantation techniques is an effective method for the excision of a tumor thrombus without sternotomy, atriotomy and CPB, avoiding possible intra- and postoperative complications in selected cases of Wilms' tumor with intraatrial thrombus extension. The case emphasises the importance of multidisciplinary communication and collaboration.

Key words: Wilms' tumor thrombus, retrohepatic vena cava disection, children.

Wilms' tumor is the most common form of renal tumor in children. ${ }^{1}$ The cases diagnosed with Wilms' tumor are identified to have tumor thrombus extending into the renal vein, vena cava and atrium by $11 \%, 4 \%$ and $0.9-1.4 \%$, respectively. ${ }^{1-3}$ Chemotherapy is the primarily suggested treatment method for those cases, and it can mostly shrink or completely eradicate the tumor thrombus., ${ }^{1,4}$ In cases with a tumor thrombus extention into the heart in which chemotherapy fails to be successful, thrombectomy may be required and is performed by opening the atrium with or without cardiovascular by-pass 
(CPB). ${ }^{5}$ In this study, we aimed to present a case of Wilms' tumor with a tumor thrombus extention into the right atrium totally excised with retrohepatic cavatomy.

\section{Case Report}

A 3.5-year-old girl was admitted with the symptom of dysuria. Evaluation revealed a mass consistent with Wilms' tumor in the middle and lower poles of the left kidney. Echocardiographic examinations of the patient showed a tumor thrombus extension into the right atrium and some pulmonary nodules which were interpreted to be metastasis. Wilms' tumor was histopathologically diagnosed by an open biopsy. Ifosfamide, carboplatin and etoposide (ICE) regimen was initiated to shrink the tumor and thrombus. After three courses of ICE treatment, imaging studies revealed that the tumor downsized significantly, and the pulmonary nodules disappeared; however, the tumor thrombus extension into the atrium persisted. The $9.1 \times 1.1 \mathrm{~cm}$ tumor thrombus was found to be fibrotic on the magnetic resonance imaging scan of the patient (Fig. 1). Therefore, nephroureterectomy along with excision of the tumor thrombus from the inferior vena cava was planned. The operation was performed by one pediatric urologist, one pediatric surgeon with liver transplantation experience and one cardiovascular surgeon. Intraoperative continuous trans-esophageal echocardiography (TEE) was performed (Fig. 2). Following the dissection of the entire renal hilum except for the renal vein, the renal vein was opened vertically, and the thrombus was dissected from the point of attachment to the tumor. Then, the suprarenal and retrohepatic vena cava were exposed by dissecting and ligating all short hepatic veins and completely mobilizing the right lobe of the liver. Inferior vena cava was completely exposed, and vascular control was achieved with snares below the renal veins and at the infrahepatic level right below the hepatic vein conjunction of the inferior vena cava at the subdiaphragmatic level to provide adequate hepatic in- and outflow during the course of surgery to prevent total vascular exclusion and thus hepatic ischemia (Fig. 3). Vertical cavatomy at the retrohepatic level was performed. Initially, the distal end of the thrombus was dissected out from the caval conjunction of the renal vein and taken out from the renal vein stump and the distal vena cava thus dissecting the thrombus cranially using endarterectomy spatula to the level of hepatic vein conjunction providing constant traction finally excising the atrial end of the thrombus (Fig. 4). Care was taken to communicate with the anesthesiologist to synchronize respiration to eliminate the risk of air embolus. TEE confirmed complete removal of the thrombus from the atrium; Vena cava was then repaired. There was no need for blood transfusion during the operation. The patient,

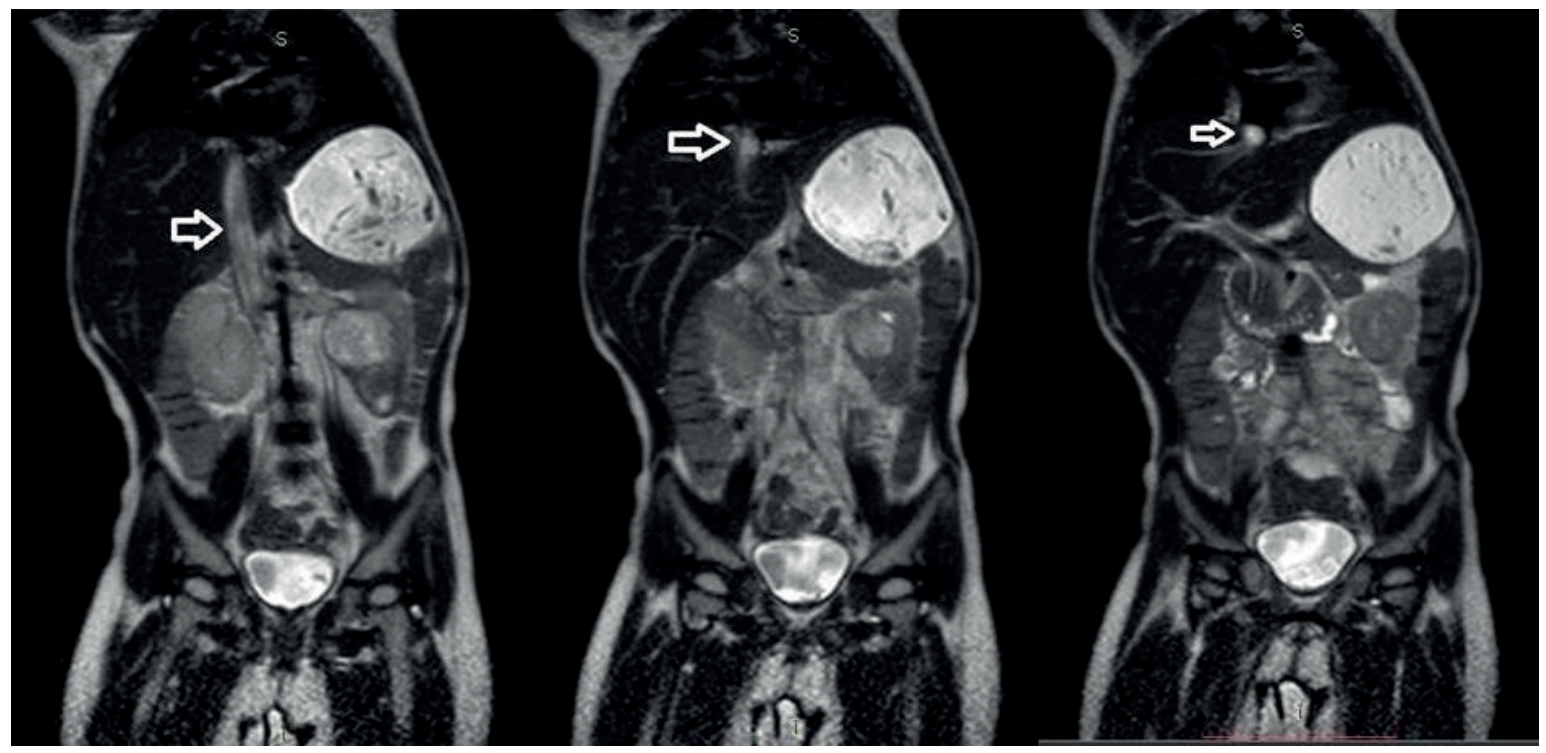

Fig. 1. MR images of thrombus 


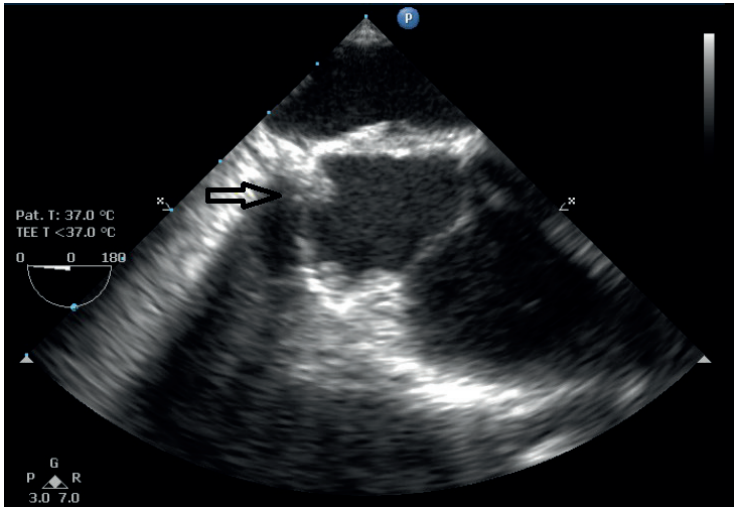

Fig. 2. Thrombus was seen in peroperative transoesophageal echocardiogram

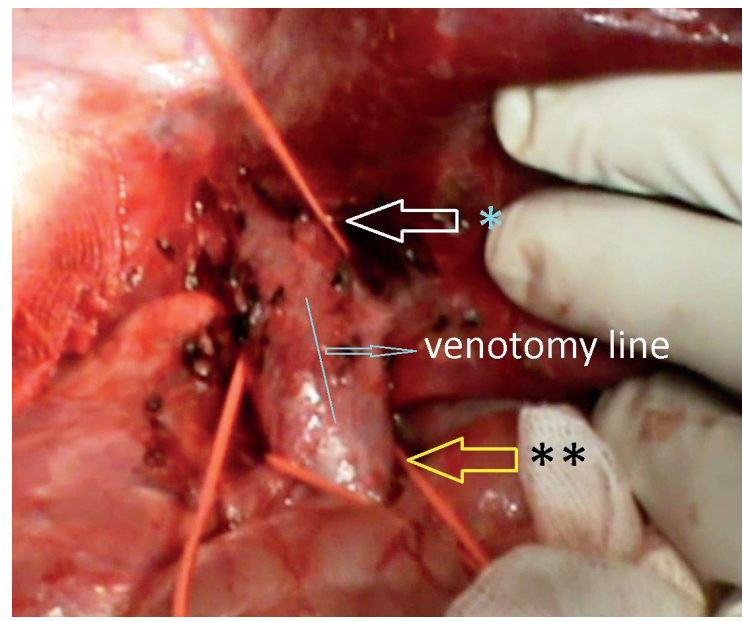

Fig. 3. Retrohepatic vena cava was demonstrated between renal vein and hepatic vein level.

${ }^{*}$ Hepatic vein level ${ }^{* *}$ renal vein level

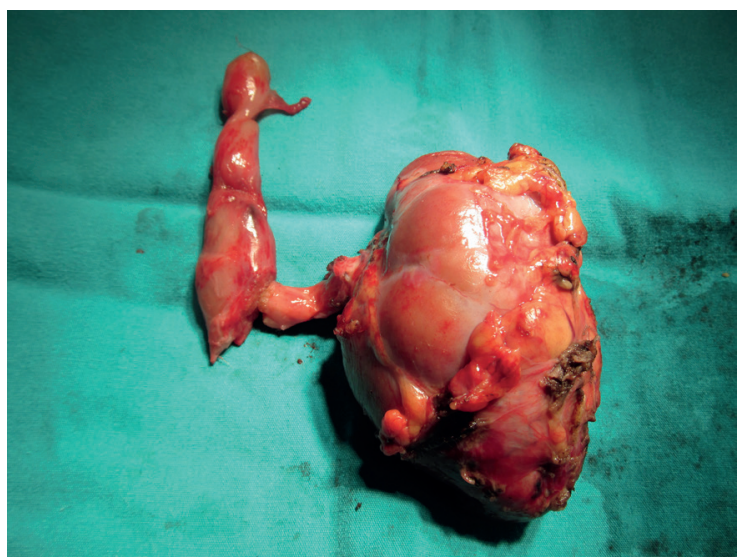

Fig. 4. Postoperative tumor and thrombus image now deceased was lost in the 8th postoperative month due to chemotherapy complications. Informed consent was obtained from the parents for publishing this case report.

\section{Discussion}

The treatment of the Wilms' tumor with intravascular extension may become a relatively complex issue. While preoperative chemotherapy provides a high rate of treatment for even large thrombi, surgical excision of persistent thrombi following chemotherapy is almost unequivocally required for definitive treatment. ${ }^{1,2,6}$ Surgery model to be utilized depends on the level of the tumor thrombus. ${ }^{7}$ For the treatment of thrombi at the supradiaphragmatic level, open heart surgery with $\mathrm{CPB}$ is a generally accepted surgical approach. ${ }^{8}$ This model requires sternotomy, atriotomy and systemic anticoagulation; and may cause complications such as hemorrhage, systemic inflammatory response syndrome (SIRS), pulmonary complications and neurological dysfunctions, although they occur less frequently today. ${ }^{6}$ Various methods have been defined to avoid these complications; however they are not fully employed in routine practice. ${ }^{6,9}$ Lodge et al. ${ }^{6}$ managed to control the intrapericardial vena cava with minimal sternotomy and excise the tumor thrombus without CPB. Another method defined for thrombi at the intrahepatic level in cases with renal cell carcinoma suggests the mobilization of the right lobe of the liver using transplantation techniques and exposal of the anterior face of the vena cava. ${ }^{10}$ In our case, the anterior face of the vena cava was exposed following retrohepatic dissection carried out by a pediatric surgeon experienced in liver transplantation, then the tumor thrombus extending into the atrium was excised by a cardiovascular surgeon. CPB device was kept operational for other complications that may have occured during surgery.

In conclusion, total exposure of the retrohepatic and subdiaphragmatic vena cava using transplantation techniques is an effective method for the excision of a tumor thrombus without sternotomy, atriotomy and $\mathrm{CPB}$, avoiding possible intra- and postoperative complications in selected cases of Wilms' 
tumor with intraatrial thrombus extension. The case emphasises the importance of multidisciplinary communication and collaboration.

\section{REFERENCES}

1. Lall A, Pritchard-Jones K, Walker J, et al. Wilms' tumor with intracaval thrombus in the UK Children's Cancer Study Group UKW3 trial. J Pediatr Surg 2006; 41: 382-387.

2. Nakayama DK, Norkool P, deLorimier AA, O'Neill JA Jr, D'Angio GJ. Intracardiac extension of Wilms' tumor. A report of the National Wilms' Tumor Study. Ann Surg 1986; 204: 693-697.

3. Wiener JS, Coppes MJ, Ritchey ML. Current concepts in the biology and management of Wilms tumor. J Urol 1998; 159: 1316-1325.

4. Oberholzer HF, Falkson G, De Jager LC. Successful management of inferior vena cava and right atrial nephroblastoma tumor thrombus with preoperative chemotherapy. Med Pediatr Oncol 1992; 20: 61-63.

5. Stewart JR, Carey JA, McDougal WS, Merrill WH, Koch MO, Bender HW Jr. Cavoatrial tumor thrombectomy using cardiopulmonary bypass without circulatory arrest. Ann Thorac Surg 1991; 51: 717-721; discussion 721-722.
6. Lodge AJ, Jaggers J, Adams D, Rice HE. Vascular control for resection of suprahepatic intracaval Wilms' tumor: Technical considerations. J Pediatr Surg 2000; 35: 1836-1837.

7. Thompson WR, Newman K, Seibel N, et al. A strategy for resection of Wilms' tumor with vena cava or atrial extension. J Pediatr Surg 1992; 27: 912-915.

8. Chiappini B, Savini C, Marinelli G, et al. Cavoatrial tumor thrombus: Single-stage surgical approach with profound hypothermia and circulatory arrest, including a review of the literature. J Thorac Cardiovasc Surg 2002; 124: 684-688.

9. Adams WM, Huskisson L, Gornall P, John PR. Temporary balloon occlusion of the inferior vena cava as an alternative to cardiopulmonary bypass in resection of Wilms' tumour with vena cava extension. Pediatr Radiol 1997; 27: 236-238.

10. Ciancio G, Vaidya A, Savoie M, Soloway M Management of renal cell carcinoma with level III thrombus in the inferior vena cava. J Urol 2002; 168(4 Pt 1): 1374-1377. 